\title{
Electrophysiological and positron emission studies in a patient with cortical myoclonus, epilepsia partialis continua and motor epilepsy
}

\author{
JMA COWAN, JC ROTHWELL, RJS WISE,* CD MARSDEN \\ From the University Department of Neurology, Institute of Psychiatry, and King's College Hospital Medical \\ School, and the MRC Cyclotron Unit, ${ }^{*}$ Hammersmith Hospital, London, UK
}

SUMMARY A patient is described who had a combination of stimulus-sensitive cortical myoclonus, epilepsia partialis continua, and Jacksonian motor epilepsy. He eventually required surgery because of the severity of his seizures. Electrophysiological recordings made before and during surgery, and PET scans performed before surgery identified an abnormal area of cerebral cortex in the postcentral parietal region. It is suggested that the stimulus-sensitive myoclonus arose because input into this region from peripheral sensory afferents produced an abnormal discharge which was fed forwards via cortico-cortical connections to the precentral motor cortex, to produce a reflex muscle jerk. The epilepsia partialis continua may have been caused by spontaneous discharges arising in the same region of parietal cortex. Both forms of jerking disappeared after resection of this part of the cortex.

Much has been written on the subject of human myoclonus, especially that arising in the cerebral cortex. ${ }^{1}$ Patients with cortical myoclonus frequently exhibit abnormally large cortical sensory evoked potentials (SEPs) and, with back-averaging techniques, it is often possible to detect a time-locked potential in the electroencephalogram (EEG) over the contralateral sensorimotor cortical area preceding spontaneous jerks. Such neurophysiological investigations usually have had to rely upon SEPs and EEGs recorded from scalp electrodes, which may not provide accurate localisation of the source of abnormality. This report concerns a patient with stimulus-sensitive cortical myoclonus, epilepsia partialis continua, and Jacksonian motor epilepsy, who eventually came to surgery because of the severity of his seizures. This provided a rare opportunity to correlate findings from direct cortical recording with those of scalp EEG, both before and after resection of an area of the post-central parietal cortex identified as the abnormal site. This area was shown to be metabolically abnormal, prior to surgery, by positron emission tomography, even though computed tomographic (CT)

Address for reprint requests: Professor CD Marsden, Institute of Psychiatry, De Crespigny Park, London SE5 8AF, UK.

Received 17 May 1985. Accepted 23 July 1985 scans showed no abnormality other than atrophy The opportunity was also taken to examine long latency stretch and cutaneous reflexes after excision of this area of cortex.

\section{Case report}

PM, a right handed 20-year-old male was referred by Dr M Yealland, in February, 1982 because of intractable epilepsy. There was no family history of neurological illness. His birth and early history were normal. At the age of 9 years he began to suffer from seizures. These commenced with a strange feeling in the left upper limb which then started to shake. Gradually, the shaking spread to involve the rest of the limb and then the left side of the face, the left side of the trunk and the left lower limb. Each seizure lasted about one to two minutes. He did not lose consciousness, but was incontinent of urine during attacks in the first few years. The seizures continued at a frequency of about four to eight per week. At the time of presentation, each attack lasted for about one and a half minutes with the left upper and left lower limbs feeling "numb" for about an hour afterwards, during which time he would feel sleepy. There had been no response to treatment with phenytoin, carbamazepine, phenobarbitone, primidone, valproate or clonazepam, all given to the point of toxicity. The fits became more frequent on occasions and he had to be admitted to hospital once in $\mathbf{1 9 8 0}$ and twice in both 1981 and 1982, because of repeated seizures, virtually amounting to status epilepticus.

In 1978 , he began to notice increasing clumsiness when 


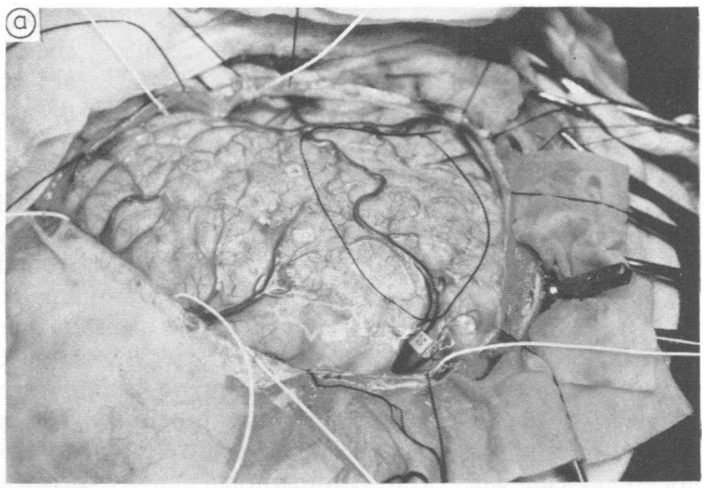

(b)
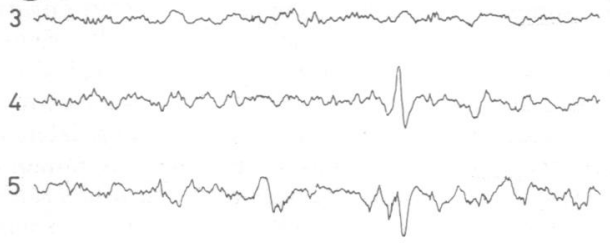

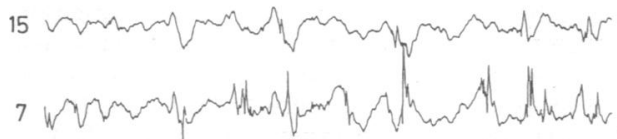

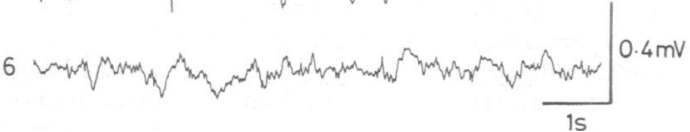

Fig 1 A. View of the operative field. The anterior aspect of the wound is on the left, and the superior aspect is at the bottom of the photograph. The right frontal, central and parietal regions can be seen through the craniotomy. The first area to be resected is outlined by the black thread. Small letters placed on the pial surface of the brain indicate the approximate extent of the motor cortex, being the lowest threshold points from which contralateral movements could be elicited by electrical stimulation. $B$. Section of intra-operative corticogram taken with the patient conscious. The traces are unipolar records from ball electrodes on the pia referred to a large metal clip on the skull. Numbers at the end of each trace indicate the electrode positions in the diagram of figure 7.

Position 7 is the point of maximal spontaneous spike activity.

using the left hand and forearm. In the middle of 1980 , he developed a continual twitching of the left fingers and hand which was always present, except during sleep, or after a flurry of seizures. Because of both the clumsiness and the twitching, he had to give up work as a trainee gardener, in 1981. Six months before his first admission to this department, he developed a twitch of the left side of the mouth which was similar to, and was present simultaneously with that of the fingers. On admission, he complained of left sided weakness.

On examination, he was alert and orientated. His speech was slow, deliberate and slurred, but there was no dysphasia. There was continual twitching of the left side of the mouth and jerks of the fingers of the left hand. The left hand and forearm would jerk in response to a variety of different stimuli, including light taps to the finger pads, electrical shocks of the digital nerves, and tendon taps to the finger flexor and extensor muscles. Limb bulk, tone and power were normal. There was impairment of position and two-point discriminatory sensations in the left fingers and a slight decrease of light touch and pin-prick sensations in the whole of the left hand.

Electroencephalograms showed gross deficiencies of fast rhythms over the right central and parietal areas. In addition, there was an excess of slow activity on the right, especially in the sylvian-central area. Multiple spike foci were seen on the right side, frequently in the centralparasaggital, mid-central, sylvian-central and mid frontal areas, and occasionally in the right temporal region. There were no left sided spikes. A normal response to photic stimulation was evident, bilaterally.

Skull radiographs were normal. CT scans with contrast showed atrophy of the right hemisphere with a prominent cisterna magna. Right carotid angiography was normal. Positron emission tomographic (PET) scans and detailed neurophysiological studies were performed and are detailed below.

On psychological testing, the short Wechsler Adult Intelligence Scale (WAIS) showed a verbal IQ of 84, a performance IQ of 79 and a full-scale IQ of 81 . No firm conclusions could be made as to whether or not a focal brain disturbance might be responsible for the low scores.

Because of the inability of anti-convulsant drugs to control his disabling seizures, and their focal origin, it was decided to proceed to electrocorticography with a view to excision of the focus so defined. A two-stage operation was performed by Mr C Polkey. During the first stage, a right temporo-parietal craniotomy was performed under general anaesthesia. Three days later, the second stage was undertaken with the patient awake. Omnopon and atropine had been given the night before, as premedication. Electrocorticography (Dr M Driver), identified the abnormal discharges as arising mainly from the right parietal lobe (for details see results). Following electrocorticography, general anaesthesia was induced. A piece of parietal cortex, measuring $6 \mathrm{~cm} \times 4 \mathrm{~cm} \times 3 \mathrm{~cm}$ was removed (fig 1). Because of continued spiking seen on the EEG, a further $3 \mathrm{~cm} \times 2 \mathrm{~cm}$ $\times 1 \mathrm{~cm}$ area was excised from the superior margin of the initial resection line. The tissue taken felt tough and abnormal. After the resections, there was still spontaneous spiking from a pre-central lead (position 4, fig 7). Because this area felt normal and had a normal reaction to cortical stimulation, it was decided not to resect it. A small piece of the excised tissue was placed in glutaraldehyde for electron microscopy and the rest was placed in formalin.

The specimens were examined by Dr B Dunkley. The grey-white junction was softened, granular and yellowish in two different areas: Several blocks were taken for light microscopy and all showed similar changes. The cortex was reduced to an outer zone of densely gliotic tissue of varying depth, containing varying numbers of neurons, and an inner zone of loose astrocytic fibrils and prominent capillary blood vessels, but no neurons. Many gemistocytic astrocytes were present in this inner zone and occasional nerve cell bodies were seen remaining in the "spongy" astrocytic mesh- 
work. The white matter showed fairly good preservation of myelin staining, but here too there was astrocytic gliosis. The leptomeninges were thickened by fibrosis and were adherent to the surface of the cortex. The blood vessels of the meninges and of the cerebral parenchyma appeared to be normal. There was no evidence of inflammation. Electron microscopy did not reveal any viral particles.

The patient made a good recovery from the operation, having only one seizure on the first post-operative day, during which the head and eyes turned to the left. There were also a few short-lasting episodes of twitching of the left thumb, but this then disappeared. Carbamazepine and clonazepam were continued.

The operation, however, resulted in further deficit. There was a left inferior quadrantanopia, a mild weakness of the left upper and lower limbs when tested against resistance, diminution in pin and touch appreciation in the left hand and forearm and marked worsening of position sense in the left hand, wrist and elbow. It was now difficult to maintain a constant position with the left hand, even with visual feedback. Neuropsychological assessment revealed significantly lower scores on tests of visual constructive ability and on the WAIS than pre-operatively.

In the eighteen months since the operation, the spontaneous twitching of the left upper limb has returned on occasions, but is absent almost all of the time. The seizures have been as frequent as before, but have not involved as wide an area of the body, and there have been no further episodes of status epilepticus or severe fits. The left upper limb reflexes have become brisk. The post-operative reduction of pinprick and touch sensations have recovered, but the visual and proprioceptive defects have remained, unaltered. The patient was able to return to the gardening job that he had had to leave because of the seizures.

\section{Methods}

\section{Physiological testing}

Neurophysiological studies included routine EEG polymyography, back-averaging of the EEG activity preceding spontaneous jerks, somatosensory evoked potentials, muscle stretch reflexes and cutaneous reflexes.

Preliminary EMG recordings were made from up to eight muscles that were involved in the myoclonic jerks. From these records, the muscle with the most well-defined and regular EMG burst was chosen to trigger collection of data for back-averaging. Each single trial was collected separately and was later averaged. In this way, any record showing movement artifact or a poor trigger point could be rejected on visual examination.

The EEG was recorded using $1 \mathrm{~cm}$ diameter silver/silver-chloride disc electrodes. Similar electrodes were placed over the cervical vertebrae, at Erb's point and on the medial aspect of the arm, $10 \mathrm{~cm}$ above the elbow over the median nerve, for somatosensory evoked potential recording, and $2-3 \mathrm{~cm}$ apart over the bellies of muscles studied for EMG activity. The signals were processed and pre-amplified with Devices 3160 pre-amplifiers and were amplified using Devices 3120 amplifiers. Filters were set $3 \mathrm{~dB}$ down at $80 \mathrm{~Hz}$ (high-pass) and $2.5 \mathrm{kHz}$ (low-pass) for EMG and shortlatency somatosensory evoked potential recording, and at $0 \cdot 16 \mathrm{~Hz}$ (high-pass) and $2.5 \mathrm{kHz}$ (low-pass) for EEG and long-latency somatosensory evoked potential recording. EMG signals were rectified or smoothed, depending on the requirements of individual studies. The resulting signals were stored on paper, on magnetic tape using a Racal tape recorder running at three and three quarter inches per second, or on floppy discs using a DEC PDP-12 computer, running programmes written by Mr HB Morton.

Somatosensory potentials were evoked either by mixed nerve stimulation of the median nerve at the wrist, or by cutaneous nerve stimulation using ring electrodes around the index finger. A Devices 3073 constant voltage stimulator was used. Stimuli were large enough to just cause visible movement of the fingers, when given at the wrist, or to give rise to a solid, but not uncomfortable tapping feeling, when delivered to the fingers (an intensity approximately twice sensory threshold). In both cases, stimuli were timed so as not to coincide with electrocardiographic activity. Because sensation was reduced in the left hand, in order for the stimulus to be appreciated, the shocks applied to the left fingers were of a greater voltage than those applied to the right. Short latency spinal and cortical potentials were evoked by stimuli given at approximately $3 \mathrm{~Hz}$ and recorded with a sampling frequency of $5 \mathrm{kHz}$ per channel. Long latency somatosensory evoked potentials were evoked at random intervals every $2-3.5$ seconds and were recorded with a sampling frequency of $1 \mathrm{kHz}$ per channel. There is some difficulty in labelling the components of the somatosensory evoked potential in patients with grossly abnormal potentials. In this paper, as before, ${ }^{2}$ the major components were designated by polarity and sequence (N1, P1, N2 etc), to avoid equating any of the myoclonic potentials with those seen in normal individuals (N20, P25/P30, N35).

Stretch reflexes were evoked in the flexor pollicis longus muscle by rapid extension of the interphalangeal joint of the thumb with the other related joints being securely clamped. The patient was asked to exert a small, constant torque of $0.06 \mathrm{Nm}$ by flexing the pad of the thumb against a torque motor. Every 2-3.5 seconds, the torque was suddenly increased by a factor of two for $200 \mathrm{~ms}$, or by a factor of four for $50 \mathrm{~ms}$ (in different studies), and the resulting EMG was recorded from the agonist muscles.

Cutaneous reflexes were studied using ring electrode stimulation of the ring finger at three times sensory threshold. Stimuli were given at $2 \mathrm{~Hz}$, while the patient exerted a constant contraction of his first dorsal interosseous muscle by fanning out the fingers of the hand, according to the technique of Jenner and Stevens. ${ }^{3}$ The averaged, rectified, EMG response of the first dorsal interosseous muscle to $256 \mathrm{stim}$ uli was obtained.

A ring block of the thumb was produced with $4 \mathrm{ml}$ of $1 \%$ plain lignocaine.

Intra-operative electrocorticography was performed using metal ball electrodes on the pial surface. The signals were fed into a conventional EEG machine and selected signals were recorded on magnetic tape for later analysis, using an output from the EEG machine taken after the pre-amplification stage as the input for the tape recorder.

\section{PET scanning}

The patient was studied twice pre-operatively with positron emission tomography and the oxygen-15 steady state inhalation technique, to determine regional cerebral blood 
flow (rCBF), oxygen consumption (rCMRO2) and their relationship-the fractional extraction of oxygen (rOER). ${ }^{4}$ There was an interval of one week between the studies, and on the second occasion, consecutive use was made of the oxygen-15 and fluorine-18 labelled 2-fluoro-2-deoxy-D-glucose $\left({ }^{18} \mathrm{~F}\right.$ deoxyglucose) as tracers, in an attempt to determine regional glucose metabolism (rCMRGlu), and the relationship between rCMRO2 and rCMRGlu-the metabolic ratio (rMR). ${ }^{5}$ rCMGlu was calculated using a simplified Sokoloff operational tracer equation. ${ }^{5}$ A lumped constant was incorporated into the tracer equation to define the different affinities of deoxyglucose (tracer) and oxygen (natural substrate) for the blood-brain barrier carrier molecule and intracellular hexokinase, and a value for this constant described for normal brain ${ }^{6}$ was used in this study. Regional cerebral blood volume (rCBV) was also measured on both occasions, using carbon- 11 monoxide as a tracer; rCBV provides an index of local vasodilatation, and the data was also used to correct the overestimation of rOER and rCMRO2 that is inherent in the simplified operational equation for the oxygen-15 steady-state technique as originally described. ${ }^{7}$

\section{Results}

\section{PHYSIOLOGY}

\section{a Pre-operative}

The EMG activity responsible for the spontaneous myoclonic jerks was found to be most prominent in the left first dorsal interosseous muscle, but also involved the left thenar, left finger flexor and left finger extensor muscles (fig 2). The duration of each burst was brief, lasting from 30 to $60 \mathrm{~ms}$. Two jerks sometimes occurred in rapid succession, separated by

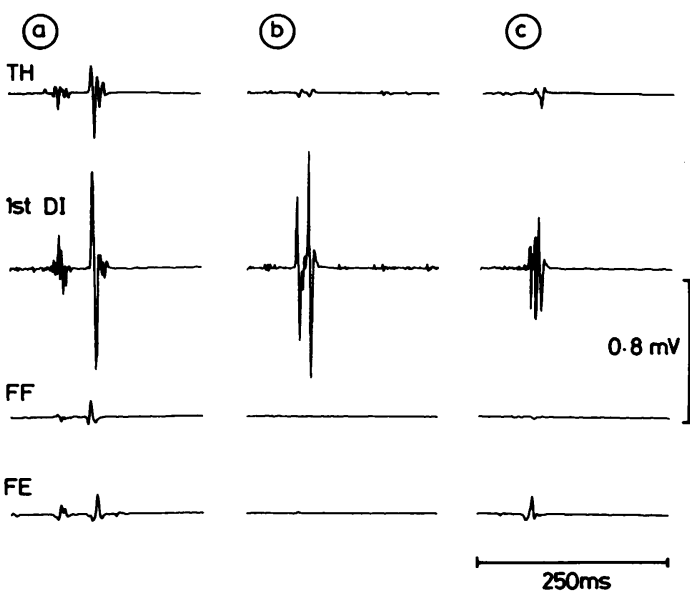

Fig 2 Sections from surface EMG records showing detail of three separate spontaneous myoclonic jerks $(A, B, C)$ in the left hand. Jerks are most prominent in the first dorsal interosseus muscle (1st DI), but also occur synchronously in thenar (TH), forearm flexor (FF) and forearm extensor $(F E)$ muscles. A rapid double jerk is illustrated in $A$.

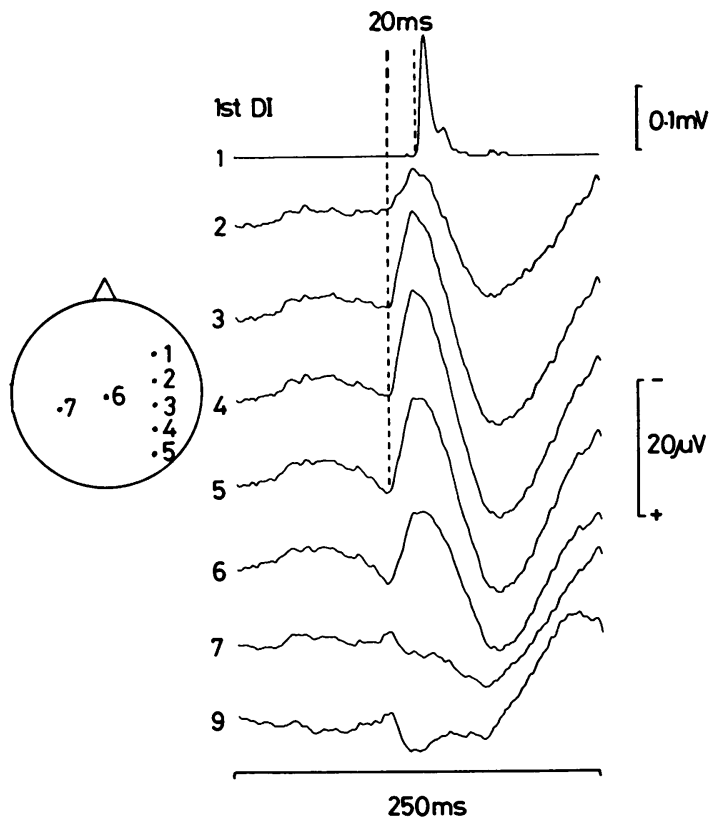

Fig 3 Back averaged (of 128 sweeps) EEG activity preceding spontaneous myoclonic jerks in the left first dorsal interosseus muscle (1st DI). A large positive-negative wave precedes the onset of EMG activity by about $20 \mathrm{~ms}$. Electrode spacing $2 \mathrm{~cm}$. Electrode 3 is $7 \mathrm{~cm}$ lateral to the vertex. Linked mastoid reference.

an interval of less than $30 \mathrm{~ms}$. The frequency of the myoclonus ranged from less than $1 \mathrm{~Hz}$ to approximately $5 \mathrm{~Hz}$. At first sight, the activation of the individual muscle groups in each jerk appeared to be approximately synchronous. However, closer examination showed that there was a slight "jitter" of about $4 \mathrm{~ms}$ between the onset of activity in the first dorsal interosseous and the other muscles. Because of this, the intrinsic hand muscles were sometimes activated before the forearm muscles and sometimes after. Back-averaging the EEG activity preceding spontaneous jerks revealed a positive-negative wave, the positive peak of which preceded the onset of EMG activity in the first dorsal interosseous muscle by about $20 \mathrm{~ms}$. This positive-negative wave was largest over the somatomotor hand area of the right hemisphere where it measured $17 \mu \mathrm{V}$ (fig 3).

Myoclonic jerks could also be provoked by electrical stimuli of the cutaneous nerve of the forefinger, or by mixed nerve stimulation of the median nerve at the wrist, in the left arm. The same stimuli also evoked large potentials over the right hemisphere. Figure 4 shows the EMG and EEG responses to electrical stimuli applied to the forefinger. Stimulation of the left forefinger produced a reflex jerk in the left 

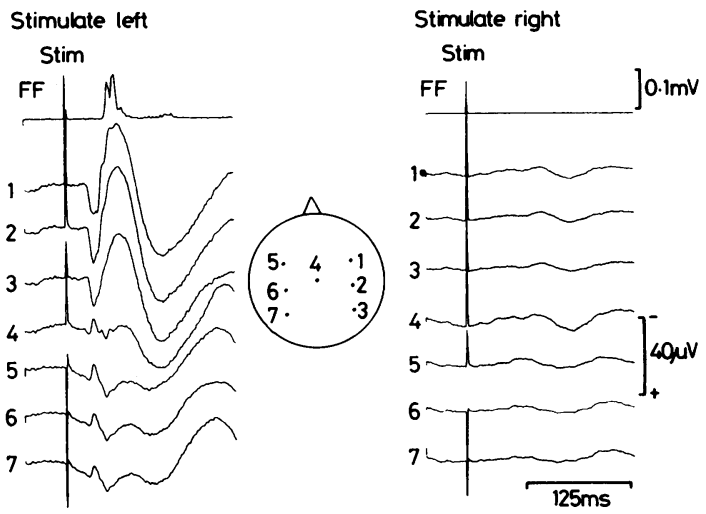

Fig 4 Pre-operative SEP following electrical stimulation (STIM) of the left and right index fingers with ring electrodes. Stimulation on the right does not provoke any muscle jerking, and the SEPs can barely be distinguished at this recording gain. Stimulation of the abnormal left hand caused a giant SEP from the right hemisphere, and a subsequent myoclonic jerk is evident in the EMG recording from the finger flexors $(F F)$. The onset of the EMG burst follows $20 \mathrm{~ms}$ after the PI peak of the SEP. Traces are the average of 128 sweeps. Electrode spacing $4 \mathrm{~cm}$. Electrode 2 is $7 \mathrm{~cm}$ lateral to the vertex. Linked mastoid reference.

forearm flexor and hand muscles, with a latency of $50 \mathrm{~ms}$. This was preceded in the SEP, by a large P1-N2 response $(45 \mu \mathrm{V})$, maximal over the contralateral central region. The P1 peak had a latency of $30 \mathrm{~ms}$ which was some $20 \mathrm{~ms}$ prior to the reflex EMG response in the hand. In contrast, stimulation of the right hand produced no reflex muscle jerk and cerebral potentials over the contralateral hemisphere were barely visible when amplified by the same amount as the signals on the other side.

The short-latency SEPs, following stimulation of the median nerve at the wrist are shown in fig 5. The gains have been increased in order to demonstrate the early responses. The peripheral (axilla and Erb's point) and spinal potentials were normal following stimulation of either side. However, the large P1-N2 component over the right hemisphere is not so evident in this figure, because low frequency components have been filtered in order to display the short-latency components, and the reference electrode was placed at $\mathrm{Fz}$ rather than using linked mastoids. The N1 response occurred at the same latency as the N20 response on the normal side and was slightly smaller.

Muscle stretches also elicited myoclonic jerks, in the left forearm and hand. When the terminal phalanx of the thumb was extended, stretch reflexes were evoked in the flexor pollicis longus (FPL) muscle and a large reflex jerk was seen in the first dorsal interosseous muscle (fig 6). The response in the first dorsal interosseous muscle lasted for $65 \mathrm{~ms}$, and occurred $7.5 \mathrm{~ms}$ after the normal responses in the FPL muscle. Responses of such a size in the first dorsal interosseous are not seen following thumb stretch in normal individuals and it is presumed that the response in PM was a reflexly-evoked myoclonic jerk. It was accompanied by a large, contralateral, somatosensory evoked potential.

In order to determine whether cutaneous or muscle afferents were responsible for the reflex jerk, the left thumb was anaesthetised with a lignocaine ring block. This abolished all cutaneous sensation in the thumb and also abolished the giant SEP in the cortex following electrical stimulation of the thumb (fig 6). As in normal individuals, the stretch reflex response in the flexor pollicis longus was reduced by anaesthesia. ${ }^{8}$ Despite this, the myoclonic response in the first dorsal interosseous muscle produced by thumb stretch was relatively unaffected, as was the somatosensory response that such stimuli evoked. Hence muscle afferents were involved in the myoclonic responses, although a small contribution from cutaneous afferents innervating the skin over the stretched flexor pollicis longus muscle cannot be excluded.

\section{$b$ Intraoperative}

The pre-operative studies suggested that this patient's myoclonus was associated with abnormal discharges in the contralateral sensorimotor cortex. Intraoperative recording allowed the site of the abnormality to be defined with far greater precision. Spontaneous spike discharges were seen (fig $1 \mathrm{~b}$ ), especially at position 7, where they were largest and most frequent, but also at position 15 and, to a lesser extent at positions 4 and 5 . Intravenous sodium thiopentone was given. This caused position 6 to become the site of most frequent spiking, as well as producing the expected increase of background rhythms in all channels. Electrocorticography revealed little abnormality of the precentral cortex. The motor strip was mapped out using $50 \mathrm{~Hz}$ alternating current stimulation and was judged to be normal.

While conscious, electric shocks were given to the left index and middle fingers, at an intensity of twice sensory threshold. The resulting somatosensory evoked potentials were very similar to those seen with scalp recording (fig 7). The major deflection at electrode 6 had an onset, latency and polarity equivalent to the P1-N2 component recorded from the scalp. No $\mathrm{N} 1$ component was evident, probably because of the relatively low setting of the high frequency filters used on the EEG machine preamplifiers. The smaller potentials recorded at the other electrodes probably are due to volume conduction of the electrical activity at or near electrode 6.

The patient had no spontaneous myoclonus during 
Electrophysiological and positron emission studies in a patient with cortical myoclonus, epilepsia partialis continua and motor epilepsy

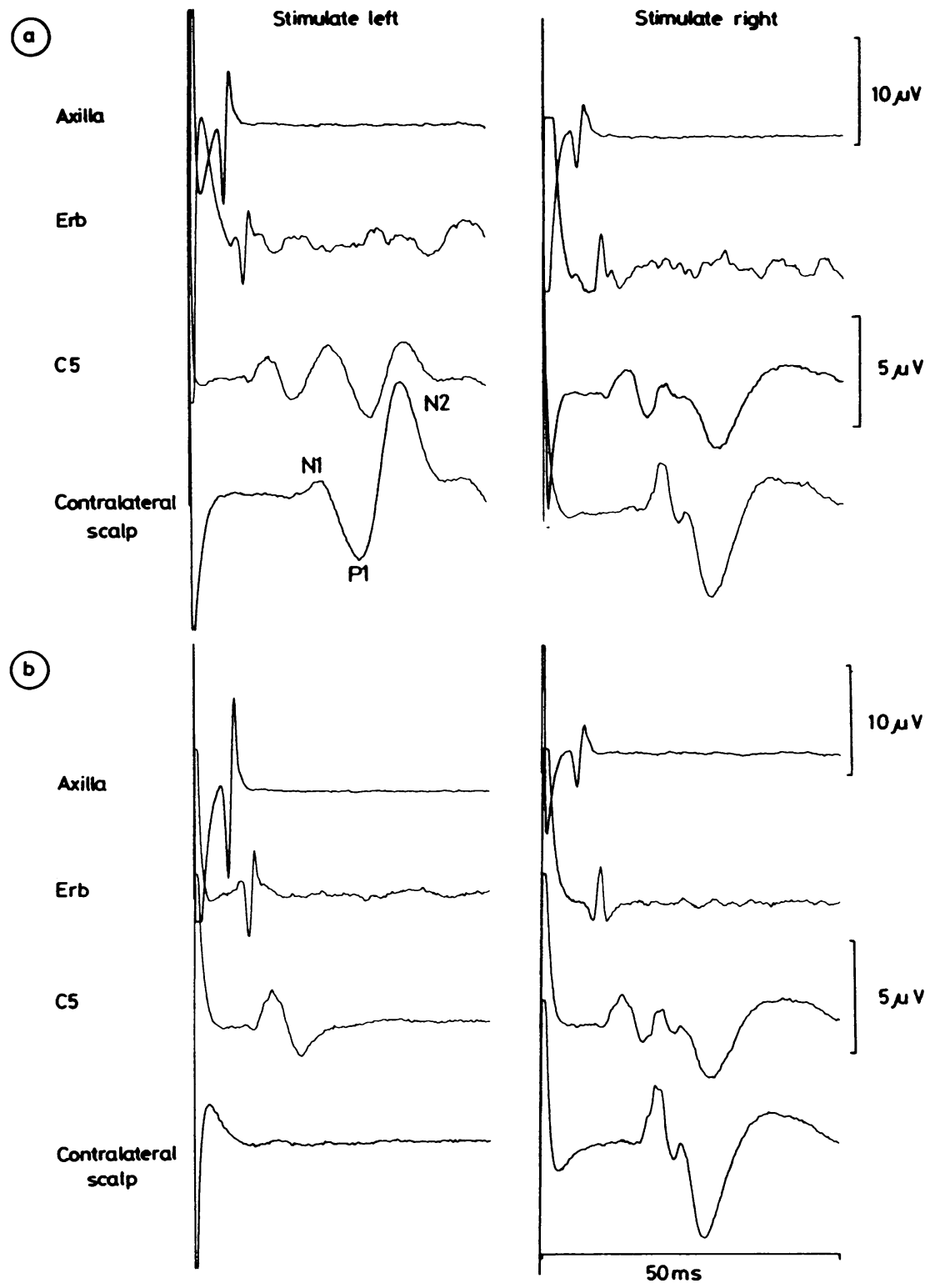

Fig 5 Short-latency SEPs following stimulation of the median nerve at the wrist. A: pre-operative, B: three weeks post-operative. Records are the average of 999 sweeps. Traces from ipsilateral axilla and Erb's point are bipolar surface recordings. Traces from an electrode over the fifth cervical spine (C5) and from contralateral sensory hand area (scalp) $(7 \mathrm{~cm}$ lateral on a line joining the external auditory meatus to a point $2 \mathrm{~cm}$ posterior to the vertex) are unipolar, referred to an electrode at Fz. Post-operatively, all cortical potentials disappear after stimulation of the left side, although the early potentials in subcortical tracings are unchanged. (The later waves in the C5 pre-operative recording are conducted from the brain via the FZ reference.) The difference in size between the axilla potentials on each side probably was due to slightly different electrode placings on each arm. The difference in pre-operative N1 amplitudes is within the normal range. Stimulus intensity was at motor threshold on each side. The giant P1-N2 component, following stimulation of the left side is not seen as clearly here as in the previous figure, because low frequency components of the EEg signal have been filtered out to display the short latency events more clearly. The $10 \mu \mathrm{V}$ calibration refers to the top trace and the $5 \mu \mathrm{V}$ calibration refers to the bottom three traces, in each section. 
Control

Electrical stimulation

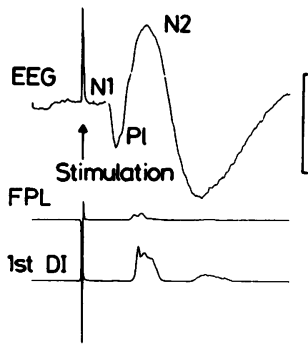

Anaesthetic

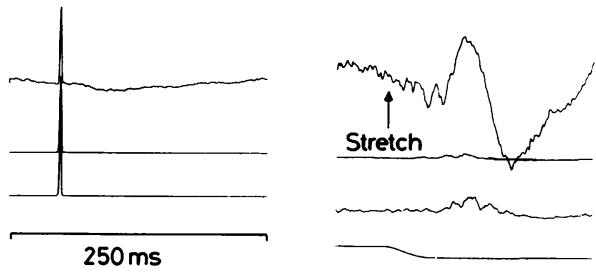

Fig 6 Effect of thumb anaesthesia produced by a local anaesthetic ring block of the proximal phalanx on the EEG and EMG responses to cutaneous or stretch stimuli applied to the left (abnormal) thumb. Records on the left show the response to electrical stimulation (STIM) of the digital nerves with ring electrodes. In the intact (control) state this evokes a large SEP over the contralateral hand area (EEG: unipolar, referred to linked mastoids) and reflex muscle jerks in flexor pollicis longus (FPL) and the first dorsal interosseus (Ist DI) muscles. With the thumb anaesthetised, all these responses disappear. Records on the right show the responses to forcible extension of the distal phalanx of the thumb by a torque motor. In the control state, traces show a large $S E P$, and reflex responses in FPL and 1st DI. The position record shows the excursion of the thumb joint. In the 1st DI, the responses to stretch are barely changed after thumb anaesthesia, whilst they are considerably reduced in FPL. Traces are averages of 128 trials.

the operation, perhaps due to the premedication, and so back-averaging could not be performed. Neither was there any reflex myoclonic response in the left hand to electrical stimulation of the fingers.

\section{c Post-operative}

Studies were repeated on several occasions during the next year. No spontaneous myoclonus and no reflex myoclonus could be found post-operatively in response to tapping, muscle stretch, or electrical shocks to the left hand, all of which had produced myoclonus pre-operatively. The N1, P1, N2 phases of the somatosensory evoked potential were found to be absent over the right hemisphere, when the stimulus was given to the left hand. Spinal potentials were preserved (fig 5). The contralateral somatosensory
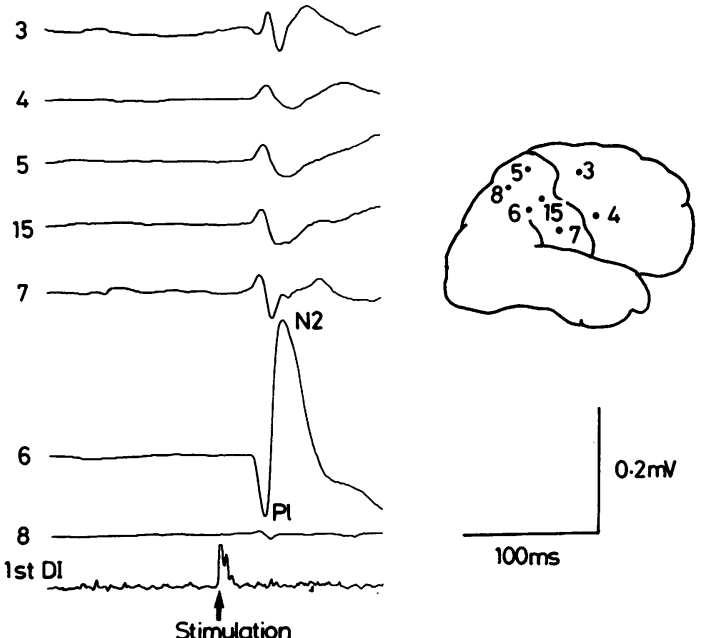

$100 \mathrm{~ms}$

Fig 7 Intra-operative SEPs following stimulation of the digital nerves of the left index finger. EEG traces are unipolar from ball electrodes resting in the pial surface as indicated diagrammatically on the right. The reference electrode was a large metal clip attached to the scalp. The bottom trace is the rectified EMG from the first dorsal interosseus (1st DI) muscle. Only the stimulus artifact (stim) is visible on the EMG record: there was no reflex muscle jerk. Average of eight sweeps. The vertical calibration applies to both EEG and $E M G$ records.

evoked potential following right hand stimulation was unchanged compared with records made before the operation.

The opportunity was also taken to investigate the effect of the removal of a well defined region of the parietal cortex on two groups of long-latency reflexes, postulated by some authors to involve transcortical pathways. When the right flexor pollicis longus muscle was stretched, normal spinal and long-latency reflexes were seen in the EMG (fig 8A). However, when the same stimulus was applied to the corresponding left sided muscle, no long-latency response could be seen. The short-latency response on the left was now twice its pre-operative size (fig 6) and was twice the size of the normal short-latency response in the right arm.

Cutaneous reflexes were studied in the first dorsal interosseous muscle, following stimulation of the index finger. The responses on the right were normal and consisted of a small short-latency E1 phase followed by the long-latency I1 and E2 responses. ${ }^{3}$ However, on the left, only the E1 response could be seen, the $\mathrm{I} 1$ and $\mathrm{E} 2$ responses being absent (fig 8B). It should be recalled that such stimulation had produced a gross myoclonic jerk on the left side before operation. 


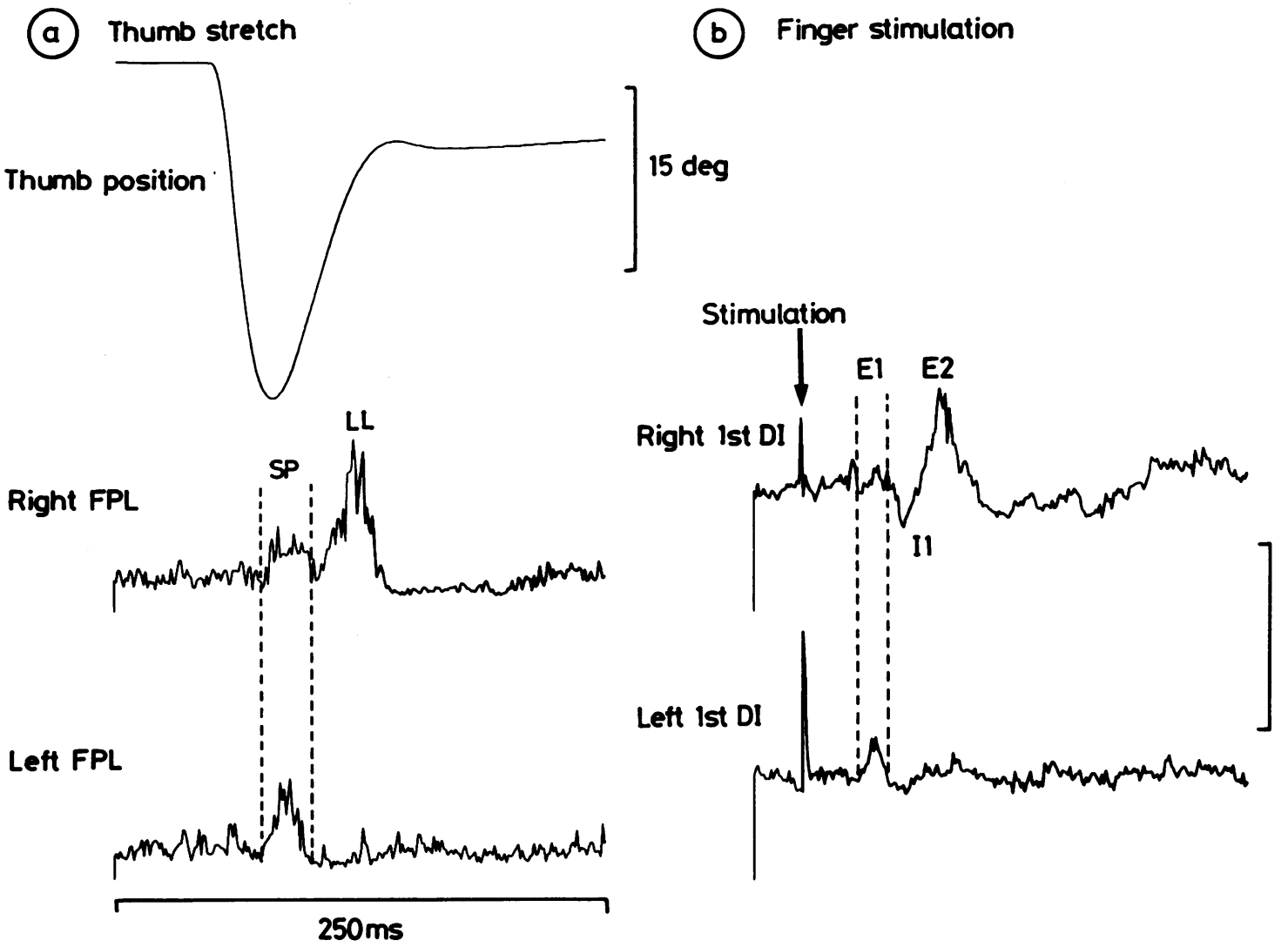

Fig 8 Post-operative comparison of reflex responses on right and left sides to: $A$, thumb stretch; and $B$, cutaneous nerve stimulation. In A, stretch reflex responses were elicited in flexor pollicis longus (FPL) by forcible extension of the distal phalanx. When this was performed on the right (normal) side, the reflex was clearly divided into a short and long latency (SP and $L L$ ) response, with onset latencies of $25 \mathrm{msec}$ and $50 \mathrm{msec}$ respectively. Thumb stretch on the left side evoked only the early muscle response. In B, electrical stimuli were given to the digital nerves of the index finger (STIM) and the cutaneous reflex responses recorded from the voluntary activated first dorsal interosseus muscle (1st DI) in the same hand. On the right, the reflex response consisted of the usual E1,II and E2 phases, whereas on the left, only the E1 was clearly visible. Rectified EMGs are the average of $32(A)$ or $256(B)$ sweeps. The vertical calibration is $0.1 \mathrm{mV}$ for $(A)$ and $0.025 \mathrm{mV}$ for $(B)$.

\section{PET SCANNING}

Positron emission tomographic scans were performed at a number of transaxial planes above the orbitomeatal (OM) line. It was evident, on inspection of the functional data, that there was high rCBF throughout the right parietal lobe, but low rCMRO2, resulting in low fractional extraction of oxygen-rOER-from the arterial blood. The region of high flow also had high $\mathrm{rCBV}$, which reflected the local vasodilatation. The values of rCBF, rCMRO2, rOER and rCBV from left and right parietal regions, $7 \mathrm{~cm}$ above the OM line, for both studies are summarised in the table; they are compared with normal values from six normal volunteers (four males, two females, mean age 35 \pm 6 years, range $22-37$ years).
It is evident that the right parietal region had much lower rCMRO2 at the times of the second study. For technical reasons, it was not possible to record the EEG during the PET studies, but it is tempting to conclude that the right parietal cortex was electrically less active during the second study; clinically, occasional jerks of the digits of the left hand occurred during both studies.

The first study included a transaxial plane at the level of the cerebellar hemispheres. rCMRO2 and $\mathrm{rCBF}$ of the cerebellum $(1.7 \mathrm{ml} / 100 \mathrm{ml} / \mathrm{min}$ and $25 \mathrm{ml} / 100 \mathrm{ml} / \mathrm{min}$, respectively) were less than half the normal values determined at the MRC Cyclotron Unit. There was no functional asymmetry between the two cerebellar hemispheres. 
Table $\quad r C B F, r C M R O 2, r O E R$ and $r C B V$ from right and left parietal regions, at $7 \mathrm{~cm}$ above the $O M$ line, from the two studies on the patient, contrasted with values from six normal volunteers

\begin{tabular}{|c|c|c|c|c|c|c|c|c|}
\hline & \multicolumn{2}{|c|}{$r C B F$} & \multicolumn{2}{|c|}{ rCMRO2 } & \multicolumn{2}{|l|}{ rOER } & \multicolumn{2}{|l|}{ rCBV } \\
\hline & $L$ & $R$ & $L$ & $\boldsymbol{R}$ & $L$ & $\boldsymbol{R}$ & $L$ & $\boldsymbol{R}$ \\
\hline $\begin{array}{l}\text { Study } 1 \\
\text { Study } 2 \\
\text { Normals }(n=6) \\
\pm \text { SD }\end{array}$ & $\begin{array}{r}41 \\
37 \\
44 \\
\pm 4\end{array}$ & $\begin{array}{l}76 \dagger \\
53^{*} \\
45 \\
\pm 4\end{array}$ & $\begin{array}{r}3.1 \\
2.7 \\
3.5 \\
\pm 0.5\end{array}$ & $\begin{array}{l}2.2^{*} \\
0.9 \dagger \\
3.6 \\
\pm 0.5\end{array}$ & $\begin{array}{r}0.41 \\
0.38 \\
0.43 \\
\pm 0.03\end{array}$ & $\begin{aligned} 0.17 \dagger \\
0.10 \dagger \\
0.43 \\
\pm 0.03\end{aligned}$ & $\begin{array}{r}3.9 \\
4.9 \\
4.2 \\
\pm 0.4\end{array}$ & $\begin{array}{r}6.3 \dagger \\
6.4 \dagger \\
4.5 \\
\pm 0.4\end{array}$ \\
\hline
\end{tabular}

rCBF ml/100 ml $/ \mathrm{min} ; \mathrm{rCMRO} 2 \mathrm{ml} / 100 \mathrm{ml} / \mathrm{min} ; \mathrm{rCBF} \mathrm{ml} / 100 \mathrm{ml}$.

*2-3 standard deviations from normal.

$\dagger 3$ or more standard deviations from normal.
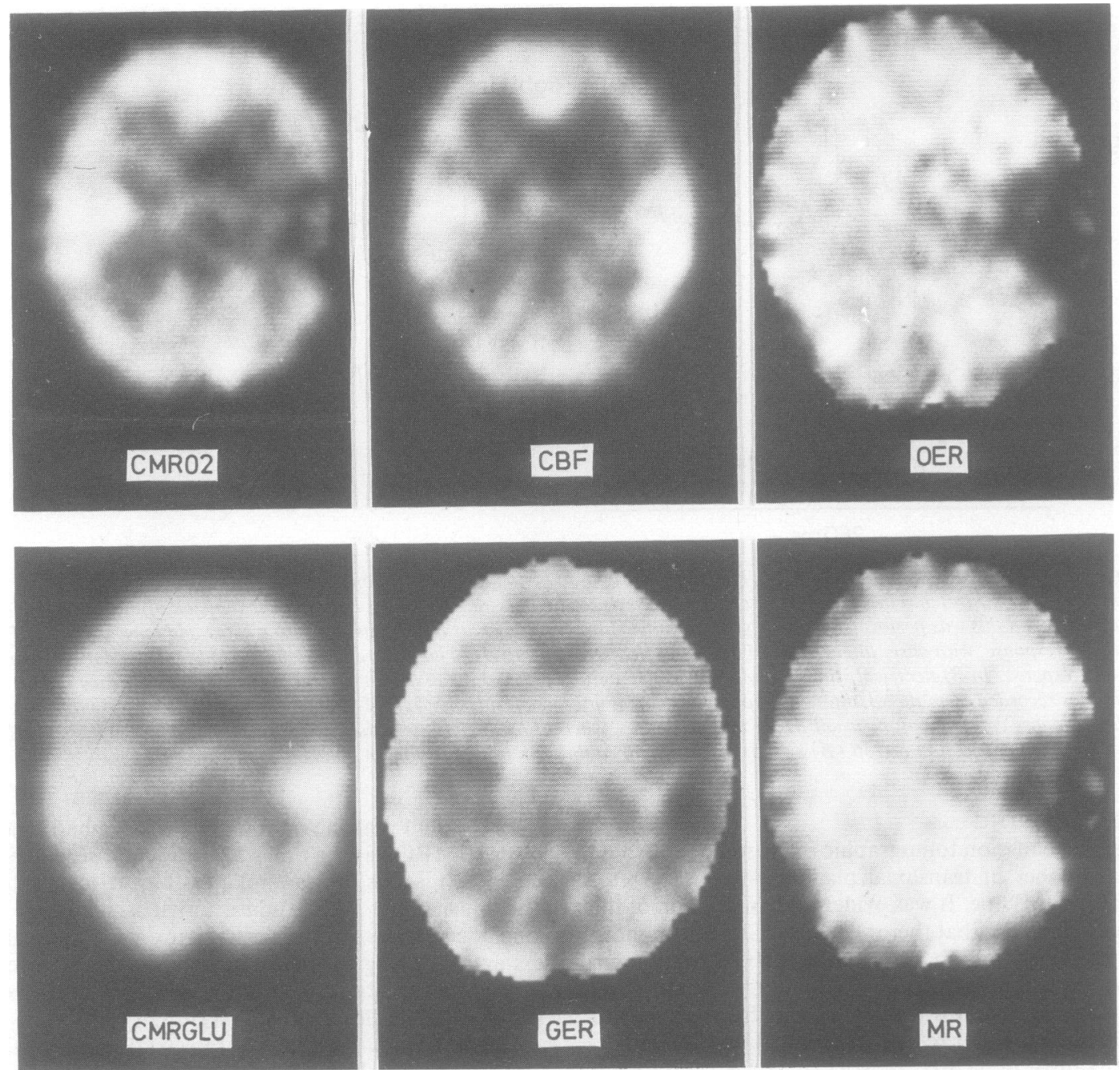

Fig 9 Functional transaxial images from the oxygen-15 and $18 \mathrm{~F}$ deoxyglucose study on the patient. The lighter the region of an image, the greater the value of a particular variable within that region. The plane depicted was $7 \mathrm{~cm}$ above the OM line. The low rCBF, rCMRO2 and rCMRGlu of the skull and scalp appear as a faint halo around the brain images of these variables. The images of the ratios appear larger because the low extracerebral signal creates patchy light-and/or dark-edge artifact when divided. $r C B F$ in the anterior part of the right parietal lobe was relatively higher than $r C M R O 2$ and $r C M R G l u$, reflected in the low $r O E R$ and $r G E R$, respectively. $r C M R G l u$ was preserved relative to rCMRO2 and therefore the rCMRO2:rCMRGlu ratio (rMR) was low in the right parietal lobe. 
Figure 9 shows grey-scaled functional images obtained from the second study at $7 \mathrm{~cm}$ above the OM line. As well as CMRO2, CBF and OER, transaxial images of CMRGlu, fractional extraction of glucose (GER) and the CMRO2:CMRGlu ratio are depicted. The low rCMRO2, high rCBF and low rOER in the right anterior parietal region are evident. As calculated, rCMRGlu was a little higher in the right parietal region than the left $(6.6 \mathrm{ml} / 100 \mathrm{ml} / \mathrm{min}$ and $6.3 \mathrm{ml} / 100 \mathrm{ml} / \mathrm{min}$, respectively), but rGER and rMR were lower. rMR in the right parietal lobe was one third that in the mirror region.

\section{Discussion}

The case reported here is a rare example of a patient with cutaneous and stretch reflex myoclonus of the left arm; regular, repetitive, continual, spontaneous myoclonus (epilepsia partialis continua); single partial seizures; and repeated partial seizures which required to be treated as status epilepticus.

There were a number of neurophysiological similarities between the epilepsia partialis continua and the cortical reflex myoclonus which suggest that both forms of jerking were manifestations of the same underlying deficit. Thus, the muscles involved, the duration of the EMG jerks and the presence of a large, contralateral, positive-negative potential in the EEG some 20 ms prior to the EMG burst were similar whether the jerks occurred spontaneously or whether they were evoked by somatosensory stimuli. Indeed, both forms of jerking disappeared after resection of the same area of the cortex. As in other cases, ${ }^{1}$ the N1 phase of the SEP was normal on the affected side of this patient, whilst later waves (P1-N2) were enlarged. The N1 phase probably corresponds to the N20 potential seen in normal subjects, which represents arrival of the afferent volley at the cortex. If this is so, then the large later wave may represent abnormal cortical processing of normal afferent inputs.

Although indicating the diversity of pathology that can give rise to the condition, previous reports of epilepsia partialis continua have tended to emphasise that an abnormality of the motor cortex is responsible for the disorder (see Thomas et al ${ }^{9}$ for references). Thomas et al discussed whether or not cortical or subcortical mechanisms are responsible for epilepsia partialis continua. They presented 32 cases of their own, including eight that had been examined after death and stated, "Autopsy findings in eight cases showed consistent involvement of the motor cortex or closely adjacent areas." They also marshalled other evidence in order to show that it was a motor cortical abnormality that had been responsible for the jerkings, in their patients, no matter where the pathologic lesion had been found. However, it has been argued before ${ }^{12}$ that areas of cortex other than the motor strip may be abnormal in patients with cortical myoclonus or epilepsia partialis continua.

Although the large, contralateral spike, which precedes cortical myoclonic jerks in the majority of patients, is most easily explained as the surface recording of a large paroxysmal depolarisation shift in motor cortical output cells, discharge of which is directly responsible for the myoclonic jerks, this may not always be true. Such potentials may be localised some centimetres anterior or posterior to the motor cortex,${ }^{10}$ and the size of the giant SEPs may be dissociated from the size of the myoclonic jerk. ${ }^{2}$ Intraoperative monitoring revealed the state of affairs in the present patient. The abnormal area of cortex lay posterior to the central sulcus and it was from this region that large SEPs could be recorded. The same region also gave rise to spontaneous spike discharges. However, the site of maximal spontaneous spike recording (electrode no 7, fig 1) was different from the site which giant somatosensory potentials could be evoked (electrode no 6). Both sites, however, were in the right parietal lobe and were within a few centimetres of each other. Perhaps the whole region was electrically unstable and this instability caused one area to respond in an exaggerated, but limited manner when receiving stimuli from its usual input pathway, whereas the other area was even more excitable and so tended to discharge either spontaneously, or else in response to otherwise undetectable stimuli. The resulting abnormal parietal cortical activity, via cortico-cortical connections between pre- and postcentral areas of cortex, may well have "driven" a physiologically normal motor cortex to discharge, and so produce visible muscle jerks. In this way, both reflex and spontaneous myoclonus could be explained. ${ }^{1112}$ Furthermore, spread of the spontaneous discharges within the abnormal parietal lobe could have fed forwards to a progressively larger area of the motor cortex, and so caused the patient's Jacksonian seizures. However, after surgery this patient's reflex myoclonus and epilepsia partialis continua disappeared, but his Jacksonian seizures persisted. Perhaps the latter continued because the area of persistent spiking in precentral cortex, demonstrated at electrocorticography, was not removed.

Surgery for epilepsia partialis continua has been reported previously. ${ }^{913}$ However, it has been frontal cortex that has been removed and this, of course, has caused the myoclonus to cease, if for no other reason than that the area resected included the cortical area responsible for the production of the clinically observed abnormality, whether or not that area was in itself abnormal. Interestingly, Thomas et al ${ }^{9}$ found surgery to be unsuccessful in controlling the epilepsia partialis continua in several of their patients and per- 
haps this is further evidence that the true seizure source lay outside the area resected (the frontal lobe).

The reflex myoclonus in this patient was of special interest in view of the recent debate about the existence of transcortical long-loop reflexes in man. It has been claimed by those who originally described such reflexes ${ }^{1415}$ that, in the upper limb at least, the longlatency phase depends on a transcortical mechanism for its production. Others have disagreed with this view and have suggested that the spinal apparatus could be solely responsible for the production of both the early and late phases, whether due to its own intrinsic delays (to explain the later response), ${ }^{16} 17$ or due to a second, later signal reaching the cord via slower peripheral nerve mechanisms, such as group II afferents, ${ }^{18}$ or even to repetitive burst discharges in muscle spindle Ia afferents following stretch. ${ }^{19}$ The long-latency responses to both stretch and cutaneous stimuli were abolished by resection of only the sensory cortex and sub-cortical white matter and this strongly supports the argument that such longlatency responses depend on a trans-cortical pathway and, furthermore, that this pathway must traverse the parietal lobe. Short-latency stretch and cutaneous reflexes, which are spinal in origin, increased in size post-operatively, whereas the long-latency reflexes disappeared. This gives further weight to the argument that the long-latency responses depend upon a different pathway.

The PET scan findings were unexpected in that although the low $\mathrm{CMRO} 2$ in the right hemisphere corresponded to the atrophy seen on the X-ray CT scan, the right parietal $\mathrm{rCBF}$ was disproportionately high relative to the $\mathrm{rCMRO}$. Not only was this reflected in the low rOER values, but was also higher than normal, despite the presence of atrophy. The tracer technique employed measured capillary blood flow and, therefore, this high flow cannot be explained by arteriovenous shunts. Furthermore, there was no evidence of any capillary angioma on histopathological examination of the resected cortical specimen. It would seem likely that the abnormal electrical activity had provoked vasodilatation and a high rCBF. In this context, it is of interest that studies of global $\mathrm{CBF}$ and rCMRO2 during electroconvulsive therapy in man have demonstrated a disproportionate rise in CBF relative to the CMRO2, and animal experiments have also shown an increase in venous $\mathrm{PO} 2$ during seizures. ${ }^{20}$ The low CBF and CMRO2 of the cerebellum may have been due to several factors, including drug therapy (the patient was on carbamazepine and clonazepam at the time of study), permanent dysfunction secondary to previous drug toxicity, and cerebellar atrophy. Low cerebellar metabolism has been observed previously in a group of patients with temporal lope epilepsy.
The combined oxygen and glucose study suggests that there was non-oxidative metabolism of glucose in the right parietal region, which, in the presence of an adequate oxygen supply, implies aerobic glycolysis. Excessive lactate production, without tissue hypoxia has been demonstrated during induced seizures in animals, ${ }^{20}$ and the same observation in man is, perhaps, not unexpected. However, there are reservations about the quantitative use of ${ }^{18} \mathrm{~F}$ deoxyglucose as a tracer in the study of epilepsy. Deoxyglucose has a greater affinity for the blood-brain carrier molecules than glucose, but has less affinity for hexokinase. If the rate limiting step for hexose phosphorylation shifts from the hexokinase-catalysed reaction to transport of substrate from plasma to tissue, the value of the lumped constant, which describes the differential rate of phosphorylation of deoxyglucose and glucose, will increase. ${ }^{21}$ Status epilepticus has been demonstrated to be associated with a reduction in tissue glucose, ${ }^{20}$ implying that under these pathophysiological conditions, blood-brain carrier transport becomes limiting. Therefore, the use of a "normal" lumped constant in this study for the calculation of rCMRGlu in all regions may have resulted in an overestimation of the degree of non-oxidative metabolism of glucose in the right parietal lobe. Recently, a method of quantifying the value of the lumped constant regionally, using an additional tracer study, has been suggested, ${ }^{22}$ and this may permit more certain estimates of rCMGlu in seizure disorders.

Nevertheless, despite uncertainties about ${ }^{18} \mathrm{~F}$ deoxyglucose tracer modelling, this is the first study to demonstrate uncoupling between regional oxygen supply and demand, and a possible regional disturbance of normal oxidative metabolism of glucose, in focal epilepsy in man.

The histology was most unusual. Neuronal loss and gliosis have been reported in the brains of those who have suffered from epilepsy. These changes have been ascribed to the effects of the seizures, ${ }^{23}$ but there are significant differences between these changes and those seen in the patient reported here. In the former cases, the neocortical changes consisted of neuronal loss mainly confined to the intermediate layers, whereas in the case presented here, the destruction was most marked in the inner layers. Also, the dense gliosis seen in the outer zone of the cortex of this patient contrasts with the relatively mild (Chaslin's) gliosis found subpially in the brains of chronic epileptics. Before operation, it was suspected that the patient had a form of chronic encephalitis, ${ }^{24}$ because of the history of normal early development, the progressive course, the restriction of clinical and EEG change to the right hemisphere and the suggestion on the PET scan of abnormal metabolism in the right parietal lobe which might have hinted at viral infec- 
Electrophysiological and positron emission studies in a patient with cortical myoclonus, epilepsia partialis continua and motor epilepsy

tion. However, no inflammatory cells were seen in the resected specimen. Thus, the cause remains unknown.

We thank PM for his interest and co-operation during the course of these investigations. The specialised equipment was designed and built by Mr HC Bertoya and Mr R Miller. This work was supported by the Medical Research Council. JCR was a Royal Society University Research Fellow.

\section{References}

${ }^{1}$ Obeso JA, Rothwell JC, Marsden CD. The spectrum of cortical myoclonus. Brain 1985;108:193-224.

${ }^{2}$ Rothwell JC, Obeso JA, Marsden CD. On the significance of giant somatosensory evoked potentials in cortical myoclonus. J Neurol Neurosurg Psychiatry 1984; 47:733-42.

${ }^{3}$ Jenner JR, Stephens JA. Cutaneous reflexes and their central nervous pathways studied in man. J Physiol (Lond) 1982;333:405-19.

${ }^{4}$ Frakowiak RSJ, Lenzie GL, Jones T, Heather JD. Quantitative measurement of regional cerebral blood flow and oxygen metabolism in man using 150 and positron emission tomography: theory, procedure, and normal values. J Comput Assist Tomogr 1980;4:727-36.

${ }^{5}$ Rhodes CG, Wise RJS, Gibbs JM, et al. In vivo disturbance of the oxidative metabolism of glucose in human cerebral gliomas. Ann Neurol 1983;14:614-26.

${ }^{6}$ Huang S-C, Phelps ME, Hoffman EJ, Sideris K, Selin CJ, Kuhl DE. Noninvasive determination of local cerebral metabolic rate of glucose in man. Am $J$ Physiol 1980;238:E69-E82.

${ }^{7}$ Lammertsma AA, Jones T. Correction for the presence of intravascular oxygen-15 in the steady-state technique for measuring regional oxygen extraction ratio on the brain: 1. Description of the method. J Cerebral Blood Flow Metabol. 1983;3:416-24.

${ }^{8}$ Marsden CD, Merton PA, Morton HB. The sensory mechanism of servo action in human muscle. $J$ Physiol (Lond) 1977;265:521-33.

${ }^{9}$ Thomas JE, Reagan TJ, Klass DW. Epilepsia partialis continua; a review of 32 cases. Arch Neurol 1977;33:266-75.
${ }^{10}$ Dawson GD. Cerebral responses to nerve stimulation in man. Br Med Bull 1950;6:326-9.

${ }^{11}$ Marsden CD, Hallett M, Fahn S. The Nosology and Pathophysiology of Myoclonus. In: Marsden CD, Fahn S, eds. Movement Disorders. London: Butterworths Scientific, 1981:96-249.

${ }^{12}$ Marsden CD. The pathophysiology of myclonus and its relation to epilepsy. Res Clin Forums 1980;2:31-46.

${ }^{13}$ Kugelberg E, Widen L. Epilepsia partialis continua. Electroencephalogr Clin Neurophysiol 1954;7:341-56.

${ }^{14}$ Marsden CD, Merton PA, Morton HB. Is the human stretch reflex cortical rather than spinal? Lancet 1973;i:759-61.

${ }^{15}$ Lee RG, Tatton WG. Motor responses to sudden limb displacement in primates with specific CNS lesions and in human patients with motor system disorders. Can J Neurol Sci 1975;2:285-93.

${ }^{16} \mathrm{Ghez}$ C, Shinoda Y. Spinal mechanisms of the functional stretch reflex. Exp Brain Res 1978;32:55-68.

${ }^{17}$ Miller AD, Brooks VB. Late Muscular responses to arm pertubations persist during supraspinal dysfunctions in monkeys. Exp Brain Res 1982;41:146-58.

${ }^{18}$ Mathews PBC. Evidence from the use of vibration that the human long-latency stretch reflex depends upon spindle secondary afferents. $J$ Physiol (Lond) 1984;348:383-416.

${ }^{19}$ Hagbarth KE, Hagland JV, Wallin EU, Young RR. Grouped spindle and electromyographic responses to abrupt wrist extension movements in man. $J$ Physiol (Lond) 1981;312:81-96.

${ }^{20}$ Siesjo BK. Brain Energy Metabolism. London: John Wiley and Sons, 1978:345-79.

${ }^{21}$ Crane PD, Partridge WM, Braum LD, Nyerges AM, Olendorf WH. The interaction of transport and metabolism on brain glucose utilisation: a re-evaluation of the lumped constant. $J$ Neurochem 1981;36:1601-4.

22 Diemer NH, Gjedde A. Autoradiographic determination of brain glucose content and visualisation of the regional lumped constant. J Cerebral Blood Flow Metab 1983;3(suppl 1):S79-S80.

${ }^{23}$ Corsellis JAN, Meldrum BL. Epilepsy. In: Blackwood W, Corsellis JAN, eds. Greenfields Neuropathology, 3rd ed. London, Edward Arnold, 1976:771-96.

${ }^{24}$ Rasmussen T. Chronic Epilepsy and Encephalitis. Applied Neurophysiology 1978;41:1-12. 